\title{
Observations of $\gamma$-ray Bursts and Solar Flares with Granat
}

O. Terekhov ${ }^{1}$, R.A. Sunyaev ${ }^{1}$, D. Denisenko ${ }^{1}$, A. Tkachenko $^{1}$, C. Barat ${ }^{2}$, J.-P. Dezalay ${ }^{2}$, R. Talon ${ }^{2}$, N. Lund ${ }^{3}$, S. Brandt ${ }^{3}$, A.J. Castro-Tirado ${ }^{3}$

${ }^{1}$ Space Research Institute (IKI), Profsoyuznaya 84/32, 117810 Moscow, Russia

${ }^{2}$ Centre d'Etude Spatiale des Rayonnements B.P. 4346, F-31029 Toulouse, France

${ }^{3}$ Danish Space Research Institute, Gl.Lundtoftevej 77, DK-2800 Lyngby, Denmark

\section{Granat orbit}

The Granat observatory was launched into a high apogee orbit on 1989 December 1 by a PROTON launcher. The initial Granat orbit had an apogee close to $200000 \mathrm{~km}$ and a perigee of $2000 \mathrm{~km}$ with an orbital period of 4 days. The satellite is entering the radiation belts for a few hours every orbit. Due to orbital evolution the perigee increased and the apogee decreased with time. After about 1.5 years of operation the perigee increased up to $20000 \mathrm{~km}$. This has put the satellite completely outside of the proton radiation belts which makes the detector activation during the perigee passage negligible. Moreover, the satellite orbit is outside of the magnetosphere during the most parts of the mission. This makes such an orbit very attractive for high energy astrophysics missions which require low background level in order to achieve high sensitivity, and makes it essential for the investigation of the high energy transient phenomena such as cosmic $\gamma$-ray bursts and solar flares.

\section{Instrumentation}

The three instruments PHEBUS (6 BGO detectors situated at the different sites of the spacecraft), SIGMA (coded mask, position sensitive Na I detector with anticoincidence shield), and WATCH (rotation modulation collimatored all-sky monitor), are able to detect and investigate $\gamma$-ray bursts and high-energy solar flares in a broad energy range from $6 \mathrm{keV}$ up to $100 \mathrm{MeV}$.

$P H E B U S$ operates in the $100 \mathrm{keV}-100 \mathrm{M} \mathrm{eV}$ energy range. Its sensitivity for gamma-ray burst detection is $\sim 10^{-6} \mathrm{erg} \mathrm{cm}^{-2}$ in almost $4 \pi$ ster. The effective area of each detector is varying from 50 to $94 \mathrm{~cm}^{2}$ depending on the GRB source position in the sky. All six detectors are recording the information about the burst simultaneously after a trigger which is provided by the PHEBUS electronics in the case if two detectors simultaneously detect count rate excesses at a level of $6 \sigma$ in the $100 \mathrm{keV}-1.6 \mathrm{MeV}$ energy range. Some prehistory of the background just before the $\gamma$-ray burst is also recorded. 
The effective area of each $\mathrm{NaI}$ and CsI scintillator detector of WATCH is $45 \mathrm{~cm}^{2}$. The sensitivity of this instrument is $\mathrm{S}(6-120 \mathrm{keV}) \sim 10^{-6} \mathrm{erg} / \mathrm{cm}^{2}$. Four detector units of this instrument are mounted on the spacecraft along four tetragonal axes. The field of view of each detector is $\pi$ ster.

The SIGMA coded mask telescope is able to detect $\gamma$-ray bursts both with Cs I anticoincidence shield detectors and with the positional sensitive detector with coded mask. The high effective area of the positional-sensitive detector gives the possibility to search (with high sensitivity of $(3-8) \times 10^{-8} \mathrm{erg} / \mathrm{cm}^{2}$ ) and to investigate $\gamma$-ray bursts occurring in the $4.7 \times 4.3 \mathrm{deg}^{2}$ field of view.

\section{Cosmic $\gamma$-ray bursts}

On the basis of the data sets published in two PHEBUS catalogues (Terekhov et al. 1994, 1995), the spatial distribution of the observed GRB sources was found to strongly deviate from a homogeneous one. From 118 cosmic $\gamma$-ray bursts observed by the PHEBUS instrument a $\left\langle V / V_{\max }\right\rangle=0.366 \pm 0.012$ is derived. This clearly shows that there is deficiency of weak GRB sources.

The question about the presence of lines in $\gamma$-ray burst spectra is important for the determination of the nature of GRB sources. Using spectral information from several detectors it is possible to increase the significance of observed line features, and to discriminate against artificial lines due to scattering or absorption from nearby mechanical hardware placed on the spacecraft. Statistical analysis of large sets of $\gamma$-ray burst spectra shows that there is no evidence of statistically significant emission features in the $511 \mathrm{keV}$ region and below at the level of few percent of the continuum emission intensity (Lobachev et al. 1995).

Simultaneous observations of cosmic $\gamma$-ray bursts in different energy ranges shows that strong spectral variability during GRBs takes place. The results of the analysis of GRB920723 revealed evidence for the presence of at least four differently varying spectral continuum components.

- A soft, slowly varying component is observable between a few $\mathrm{keV}$ - few tens of $\mathrm{keV}$. This component probably is dominating the X-ray rich GRBs observed by WATCH (Castro-Tirado et al. 1994).

- Spectral analysis of the SIGMA (Pelaez et al. 1994) data as well as BATSE (Schaefer et al. 1992) results show that the energy spectra of some $\gamma$-ray bursts are more compatible with broken power law (different spectral components below and above a certain break energy) rather than with a single component law. Detailed analysis of the PHEBUS data for GRB920723 shows that both these two components are varying with time during the GRB event (Terekhov et al. 1995b).

- In addition to these three components an additional very hard power law component appears sporadically during the GRB event for very short (less than $1 \mathrm{~s}$ ) time intervals (Terekhov et al. 1995b). During its presence, such a component dominates the whole energy range above $100 \mathrm{keV}$. 
The WATCH instrument observations of $\gamma$-ray bursts show that in some cases such as GRB920723 and GRB900222, the decaying flux of soft X-ray emission in the $6-20 \mathrm{keV}$ energy range follows the GRB event during at least $40 \mathrm{~s}$ after main gamma-ray pulse with a typical duration of $10 \mathrm{sec}$ (Terekhov et al. 1993; Castro-Tirado et al. 1994). About $10 \%$ of the WATCH $\gamma$-ray bursts display Xray tails or $\mathrm{X}$-ray precursor activity. There is no preferential concentration for such $\mathrm{X}$-ray rich events (for instance to the galactic plane or galactic center). The isotropic sky distribution was found for all sets of $\gamma$-ray bursts (32 events) localised by the WATCH instrument (Castro-Tirado et al. 1994).

WATCH is able to set upper limits for the X-ray flux from any point in the sky which is in its field of view. This means that it would detect any significant $\mathrm{X}$-ray activity from $\gamma$-ray bursts which is precisely localised. The WATCH localisation accuracy is less than $1^{\circ}$ error radius. No quiescent radiation is detected neither before nor after $\gamma$-ray burst events from the $\gamma$-ray burst sources. The upper limits for the quiescent emission fluxes from GRB920723 as well as from other GRB sources are $\sim 50$ mCrab for time scales of hours and $\sim 20$ mCrab for time scales of days.

A large fraction of the cosmic $\gamma$-ray bursts detected by the PHEBUS instrument are short. About $30 \%$ of the events have durations less than $2 \mathrm{~s}$. Such events have energy spectra which are significantly harder than the energy spectra of the long $\gamma$-ray bursts. It is interesting to note that in spite of the short duration the time histories of such events are very complex. Time histories of short $\gamma$-ray bursts consist of individual flashes with duration $2-10 \mathrm{~ms}$ and with the separation between individual flashes of the order of $10 \mathrm{~ms}$. Some individual pulses have very sharp front and decay edges with characteristic times $0.2-0.4$ ms as in the case of GRB900320 (Terekhov et al. 1992).

The SIGMA telescope was pointed towards the galactic center for almost $18 \%$ of its observing time. For an object placed at the distance of the galactic center the threshold burst luminosity in the $40-90 \mathrm{keV}$ energy range is $3 \times 10^{38} \mathrm{erg} \mathrm{s}^{-1}$, thus only slightly exceeding the Eddington luminosity for a neutron star. The absence of weak GRBs from the galactic center direction indicates that either the luminosity of typical GRBs is below $10^{38} \mathrm{erg} \mathrm{cm}^{-2}$ or, alternatively, the luminosity is higher than $10^{40} \mathrm{erg} \mathrm{s}^{-1}$ (Sunyaev et al. 1993). Note that recent observations of the BATSE instrument gave detailed constraints based on the absence of the GRB source clustering to the galactic plane and LMC reported by Meegan et al. (1994) for local disk and extended halo models (Hartmann 1995).

Observations of extragalactic sources with the SIGMA telescope gave constraints for the frequency of the appearance of GRB events for different extragalactic objects in a suggestion that GRBs are of extragalactic origin. Upper limits can be derived for the density of GRB sources per one solar mass of the matter for some of the extragalactic objects (Sunyaev et al. 1993). 


\section{High-energy solar flares}

Granat carried out its observations during the solar maximum which took place in 1990-1991. During this time interval very high solar activity was observed by Granat instruments. The most interesting data on observations of high energy solar flares in the $100 \mathrm{keV}-100 \mathrm{MeV}$ energy range were obtained by the $P H E$ $B U S$ instrument. Some of these bursts have also been observed up to $15 \mathrm{MeV}$ by the SIGMA anticoincidence shield (Pelaez et al. 1992). Over 80 solar flare events with significant emission above $100 \mathrm{keV}$ were detected during 1990-1993.

About $26 \%$ of these solar flares are associated with extremely intense GOES $\mathrm{X}$-class events. Among these solar flares there are several extraordinal events with significant emission up to $100 \mathrm{MeV}$. Among the most interesting solar flares is SF900524 - a classical example of the high energy solar flare. During this flare a complex of high energy phenomena was observed which usually are associated with high energy solar flares: photons with energy up to $100 \mathrm{MeV}, \pi^{0}$ decayed photons, high energy neutrons, $2.2 \mathrm{MeV}$ line associated with deuterium synthesis on the solar surface, fast protons appeared in a very short time after the beginning of the $x$-ray event (Terekhov et al. 1993,1995c; Talon et al. 1993).

In the flare SF910125 $\gamma$-rays up to $10-16 \mathrm{MeV}$ are detected. No emission in the $65-124 \mathrm{MeV}$ energy range is found for this flare. The flux of this flare in the energy range $1-4 \mathrm{MeV}$ was by a factor of 30 lower than one for SF900524. It is interesting to note that according to GOES classification based on soft X-ray observations these two flares have nearly equal intensities: X9.3 for SF900524 and $\mathrm{X} 10.0$ for SF910125. The heliocentric angle was also not dramatically different for these flares: $81^{\circ}$ for SF900524 and $77^{\circ}$ for SF910125.

Some solar flares detected by the PHEBUS instrument have quasi-periodic pulsations of the intensity with the typical period of $\sim 10 \mathrm{~s}$. SF900524 and SF910125 also have such important feature in their light curves. The $Q$-factors (number of separate pulses with the typical period) of these two flares are different. While $Q=16$ for SF910125, it is only 4-5 for SF900524. The modulation depth of the emission can be estimated as $\sim 0.32$ for SF900524 and $\sim 0.27$ for SF910125.

Deep modulation of hard X-ray and microwave emission with a period $\sim 10 \mathrm{~s}$ was explained in terms of Alfvenic oscillations of a single flaring loop (Stepanov et al. 1992). In this model these pulsations are driven by Alfvenic oscillations of a flare loop excited due to upward motion of chromospheric evaporated plasma. According to this model one can estimate the density $n$ and temperature $T$ of the evaporated plasma as well as the flare loop magnetic field $B$ and loop length $L$. It is possible to also estimate the pulsations (Stepanov et al. 1992), and that the lengths of the loops $L$ of these two flares are nearly the same and equal to $10^{9} \mathrm{~cm}\left(L_{9} \sim 1\right)$. Based on this model it is possible to find that during SF900524 $T \approx 4.7 \times 10^{7} L_{9}^{2} \mathrm{~K}$ which is about a factor two higher than in SF910125, $n \approx 1.5 \times 10^{12} L_{9}^{3} G$ one order of magnitude higher and $B \approx 6.1 \times 10^{2} L_{9}^{5 / 2} G$ which is 4 times higher than in the case of SF910125. These differences probably play an important role in extremely unusual properties of the SF900524 solar flare during which particles were accelerated up to very high energies. 


\section{References}

Castro-Tirado A.J., Brandt S., Lund N., Lapshov I.Y., Terekhov O., Sunyaev R.A., 1994, Gamma-Ray Bursts, G. Fishman, J. Brainerd, K. Hurley (eds.), AIP Conference Proceedings 307, American Institute of Physics, New York, p. 17

Hartman D., 1995, The Lives of the Neutron Stars, NATO ASI C 450, M.A. Alpar, U. Kiziloglu, J. van Paradijs (eds.), Kluwer, Dordrecht, p. 495

Lobachev V.A., Terekhov O.V., Denisenko D.V., Sunyaev R.A., Kuznetsov A.V., Tkachenko A.Yu., Barat C., Dezalay J.-P., Talon R., 1995, Astr. Letters 21 (in press)

Meegan C., Fishman G., Wilson R., Brock M., Horack J., Paciesas W., Pendleton G., Briggs M., Koshut T., Kouveliotou C., Teegarden B., Matteson J., Hakkila J., 1994, Gamma-Ray Bursts, G. Fishman, J. Brainerd, K. Hurley (eds.), AIP Conference Proceedings 307, American Institute of Physics, New York, p. 3

Pelaez F., Mandrou P., Niel M., Mena B., Vilmer N., Trottet G., Lebrun F., Paul J., Terekhov O., Sunyaev R., Churazov E., Gilfanov M., Denisenko D., Kuznetsov A., Dyachkov A., Khavenson N., 1992, Solar Phys. 140, 121

Pelaez F., Bouchet L., Jourdain E., Niel M., Claret A., Laurent P., Lebrun F., Paul J., Terekhov O., Sunyaev R., Kuznetsov A., Denisenko D., Gilfanov M., Churazov E., Khavenson N., Diachkov A., 1994, ApJS 92, 651

Schaefer B.E., Teegarden B., Cline T., Fishman G., Meegan C., Wilson R., Paciesas W., Pendleton G., Matteson J., Band D., 1992, ApJ 393, L51

Stepanov A.V., Urpo S., Zaitsev V.V., 1992, Solar Phys. 140, 139

Sunyaev R., Churazov E., Gilfanov M., Terekhov O., D'iachkov A., Khavenson N., Kovtunenko V., Kremnev R., Claret A., Lebrun F., 1993, ApJ 402, 579

Talon R., Trottet G., Vilmer N., Barat C., Dezalay J., Sunyaev R., Terekhov O., Kuznetsov A., 1993, Solar Phys. 147, 137

Terekhov O.V., Sunyaev R.A., Kuznetsov A.V., Molchanov A.Yu., Barat C., Vedrenne G., Dezallay J.-P., Niel M., Talon R., 1992, Frontiers of X-ray Astronomy, Proceedings of the YAMADA Conference XXVIII, Frontiers Science Series - 2, Y.Tanaka, K. Koyama (eds.) Universal Academy Press, Tokyo, p. 253

Terekhov O.V., Sunyaev R.A., Kuznetsov A.V., Barat C., Talon R., Trottet G., Vilmer N., 1993, Astr. Letters 19, 163

Terekhov O.V., Lobachev V.A., Denisenko D.V., Lapshov I.Y., Sunyaev R.A., Lund N., Castro-Tirado A.J., Brandt S., 1993, Astr. Letters 19, 686

Terekhov O.V., Denisenko D.V., Lobachev V.A., Sunyaev R.A., Kovtun A.V., Kuznetsov A.V., Barat C., Dezalay J.-P., Talon R., 1994, Astr. Letters 20, 323

Terekhov O.V., Denisenko D.V., Lobachev V.A., Sunyaev R.A., Kuznetsov A.V., Tkachenko A.Yu., Barat C., Dezalay J.-P., Talon R., 1995a, Astr. Letters 21 (in press)

Terekhov O.V., Lobachev V.A., Sunyaev R.A., Kovtun A.V., Denisenko D.V., Kuznetsov A.V., Barat C., Dezalay J.-P., Talon R., 1995b, Astr. Letters 21 (in press)

Terekhov O.V. et al., 1995c, Astr. Letters 21 (in press) 